\title{
Medizinische Entscheidungen am Lebensende in der Schweiz: Beginn der zweiten Befragung
}

Im Rahmen des EU-Projektes «Medical End-of-Life Decisions: Attitudes and Practices in 6 European Countries»

S. Fischer ${ }^{a}$, G. Bosshard ${ }^{b}$, K. Faisst ${ }^{a}$, U. Zellweger $^{a}$ medizin der Universität Zürich (ISPMZ).

b Institut für Rechtsmedizin der Universität Zürich (IRMZ).

* Wissenschaftliche Expertengruppe Prof. Dr. med. Walter Bär, IRMZ; Prof. Johannes Fischer, Institut für Sozialethik der Universität Zürich; Prof. Dr. med. Felix Gutzwiller, ISPMZ; Prof. Rainer Hornung, Psychologisches Institut der Universität Zürich, Sozialpsychologie II; Prof. Dr. med. Rudolf Ritz, Mitglied der Zentralen Ethikkommission der Schweizerischen Akademie der Medizinischen Wissenschaften; Dr. Alois Tschopp, ISPMZ; Dr. med. Thomas Spuhler, Bundesamt für Statistik. a Institut für Sozial- und Präventiv-

\section{Einleitung}

Im letzten Jahr begann in der Deutschschweiz eine Studie mit dem Ziel, Einblick in die verschiedenen Situationen zu gewinnen, in welchen Ärztinnen und Ärzte Entscheidungen treffen müssen, die das Lebensende ihrer Patientinnen und Patienten betreffen [1, 2]. Die Untersuchung wird im Rahmen des europäischen Projekts «Medical End-of-Life Decisions: Attitudes and Practices in 6 European Countries» durchgeführt und dauert insgesamt drei Jahre. In der Schweiz liegt die Projektverantwortung beim Institut für Sozial- und Präventivmedizin und beim Institut für Rechtsmedizin der Universität Zürich. Eine wissenschaftliche Expertengruppe*, die sich aus Vertretern verschiedener Fachrichtungen zusammensetzt, begleitet die Studie. Das Projekt wird von der Schweizerischen Akademie der Medizinischen Wissenschaften (SAMW) und der Oncosuisse unterstützt. Die Untersuchung gliedert sich in zwei Teile, und zwar in eine «Death Certificate Study» (Todesfallstudie) und eine «Physician Study» (Ärztestudie). Die Ergebnisse sollen das Verständnis für gesellschaftliche und kontextuelle Faktoren, welche zu unterschiedlichen Sterbehilfeentscheiden führen, verbessern. Leider konnte der erste Studienteil aus finanziellen Gründen nur in der Deutschschweiz durchgeführt werden. Dank zusätzlicher finanzieller Mittel kann der zweite Teil auf die gesamte Schweiz ausgedehnt werden.

\section{Todesfallstudie}

Susanne Fischer, lic. phil. Institut für Sozial- und Präventivmedizin, Universität Zürich Sumatrastrasse 30 CH-8006 Zürich

E-Mail: susannef@ifspm.unizh.ch

Die erste Studie, deren Datenerhebung im Winter 2002 abgeschlossen wurde, verfolgt das Ziel, streng quantitative Angaben zur Inzidenz und scheidungen am Lebensende zu gewinnen. $\mathrm{Zu}$ diesem Zweck wurden 5000 Ärztinnen und Ärzte angeschrieben und zu einem konkreten Todesfall befragt. Über 3300 ausgefüllte Fragebogen wurden retourniert! Durch den ausserordentlich hohen Rücklauf von $67 \%$ ist es möglich, repräsentative Ergebnisse zu erhalten.

\section{Ärztestudie}

Die Datenerhebung der zweiten Studie wird im Herbst 2002 erfolgen. Bei dieser schriftlichen Befragung werden je rund 300 Ärztinnen und Ärzte aus den Fachgebieten Allgemeinmedizin, Anästhesie/Intensivmedizin, Chirurgie, Gynäkologie, Innere Medizin, Neurologie, Onkologie und Pulmonologie angeschrieben. Diese Untersuchung soll die erste Befragung ergänzen und vertiefen. Es sollen Aussagen über mögliche Faktoren - wie Arbeitssetting und Kontext der Sterbesituation -, welche zu Unterschieden hinsichtlich Einstellungen und Praktiken führen könnten, gewonnen werden. Dazu werden, neben soziodemographischen Angaben, Arbeitserfahrungen, Einstellungen und - anhand von Fallbeispielen - Verhaltensabsichten von Ärztinnen und Ärzten im Umgang mit Sterbenden erfasst. Die Fallbeispiele sind auf eine terminale Krebserkrankung beschränkt, da diese Situation allen involvierten klinischen Disziplinen gleichermassen vertraut sein dürfte.

\section{Datenschutz - Rechtliches} den Hauptmerkmalen von medizinischen Ent-
Das gewählte Studiendesign gewährleistet die vollständige Anonymisierung der Daten. Der Datenschutz wurde durch den kantonalen Datenschutzbeauftragten geprüft und bestätigt. 


\section{Dank}

Wir möchten uns hiermit bei allen Ärztinnen und Ärzten herzlich bedanken, die sich - trotz ihres oft hektischen Arbeitsalltags - die Zeit nahmen, den Fragebogen bzw. mehrere Fragebogen der ersten Studie auszufüllen! Um sowohl auf nationaler als auch auf internationaler Ebene repräsentative Ergebnisse zu erhalten, sind wir auch bei der zweiten Befragung auf eine hohe Teilnahme angewiesen. Die Ergebnisse sind um so wichtiger, da für die Schweiz kaum Untersuchungen über die Einstellungen von Ärztinnen und Ärzte im Sterbehilfebereich vorhanden sind und daher Daten als Grundlage für öffentliche und fachliche Diskussionen fehlen.

\section{Literatur}

1 Faisst K. Medizinische Entscheidungen am Lebensende in der Schweiz. Im Rahmen des EU-Projekts «Medical End-of-life Decisions: Attitudes and Practices in 6 European Countries». Schweiz Ärztezeitung 2001;82(25):1329-30.

2 Bosshard G. Die Debatte um die Regelung von Sterbehilfe in der Schweiz und die MELS-Studie. Primary Care 2001;1:600-4. 